\title{
ON TJURINA TRANSFORM AND RESOLUTION OF DETERMINANTAL SINGULARITIES
}

\author{
HELGE MØLLER PEDERSEN
}

\begin{abstract}
Determinantal singularities are an important class of singularities, generalizing complete intersections, which recently have seen a large amount of interest. They are defined as preimage of $M_{m, n}^{t}$ the sets of matrices of rank less than $t$. The linear algebraic structure $M_{m, n}^{t}$ gives rise to some interesting structures on determinantal singularities. In this article we will focus on one of these, namely the Tjurina transform. We will show some properties of it, and discuss how it can and how can not be used to find resolutions of determinantal singularities.
\end{abstract}

\section{INTRODUCTION}

Hypersurface singularities have in general been the starting point of singularity theory. They have some very good properties, one of the most important is the existence of the Milnor fibration Mil68. The Milnor fibration makes it possible to define the Milnor number $\mu$, which is a very important invariant. So a goal in singularity theory is to find more general families of singularities, for which it is possible to define the Milnor number. A classical example of a generalization, for which the Milnor number can be defined, is the complete intersections. Determinantal singularities are a generalization of the complete intersections, they are defined as the preimage of the set of $m \times n$ matrices of rank less than $t$ under certain holomorphic maps. They have seen a lot of interest lately, there have been several different ways to define the Milnor number of certain classes of determinantal varieties by Ruas and da Silva Pereira SRDSP14, Damon and Pike DP14] and Nuño-Ballesteros, Oréfice-Okamoto and Tomazella [NBOOT13, Ebeling and Gusein-Zade defined the index of a 1-form GZĖ09, and in addition to these the deformation theory has been studied in GR15.

In this article we study other aspects of determinantal singularities, not directly related to deformation theory, namely, transformations and resolutions. They played a very important role in GZĖ09, and the Tjurina transform, which will be one of our main subjects, was also studied intensely for the case Cohen-Macaulay codimension 2 by Frühbis-Krüger and Zach in FZ15.

We first study the Tjurina, Tjurina transpose and Nash transformations for the model determinantal singularity in section 3. This was already done in GZĖ09], but we will need this as motivation for introducing the transformations for general determinantal singularities. We also explore how these transformations are related and how they are not, and give a description of their homotopy type. We introduce the Tjurina transform (and its transpose) for general determinantal singularities in section 4 give some general properties, for example that the Tjurina transform of a complete intersection is itself, and give some methods to find the Tjurina transform. In section 5 we show that under some general assumptions the Tjurina transform or its dual is a complete intersection. This unfortunately mean that Tjurina transform

2010 Mathematics Subject Classification. 14B05, 32S05, 32S45.

Key words and phrases. Resolution of singularities, Determinantal singularities. 
is not going to provide resolutions in general. At last we illustrate in section 6 that by changing the determinantal type of the Tjurina transform of certain hypersurface singularities, we can continue the process of taking Tjurina transform, and in the end reach a resolution. Section 2 introduces the determinantal singularities and notions of transformations used throughout the article. I wish to thank Maria Ruas for introducing me to the subject of determinantal singularities and for many fruitful conversations during the preparation of this article. The author was supported by FAPESP grant 2015/08026-4.

\section{Preliminaries}

In this section we give the basic definitions and properties of determinantal singularities, and transformations we will need. We will in general follow the notation Ebeling and Gusein-Zade used in [GZĖ09].

2.1. Determinantal Singularities. Let $M_{m, n}$ be the set of $m \times n$ matrices over $\mathbb{C}$, then we define the model determinantal singularity of type $(m, n, t)$, denoted by $M_{m, n}^{t}$, to be the subset of $M_{m, n}$ consisting of matrices $A$ of $\operatorname{rank}(A)<t . M_{m, n}^{t}$ has a natural structure of an irreducible algebraic variety, with defining equations given by requiring that the $t \times t$ minors have to vanish. The dimension of $M_{m, n}^{t}$ is $m n-(m-t+1)(n-t+1)$. The model determinantal singularities are often called generic determinantal singularities as for example in [SRDSP14].

The singular set of $M_{m, n}^{t}$ is $M_{m, n}^{t-1}$ and the decomposition of $M_{m, n}^{t}=\bigcup_{i=1}^{t}\left(M_{m, n}^{i}-\right.$ $\left.M_{m, n}^{i-1}\right)$, where $M_{m, n}^{0}:=\emptyset$, is a Whitney stratification.

Let $F: U \subset \mathbb{C}^{N} \rightarrow M_{m, n}$ be a map with holomorphic entries. We say that $X:=F^{-1}\left(M_{m, n}^{t}\right)$ is a determinantal singularity of type $(m, n, t)$ if $\operatorname{codim}(X)=$ $\operatorname{codim}\left(M_{m, n}^{t}\right)=(m-t+1)(n-t+1) . X$ has the structure of an irreducible algebraic variety, with equations defined by the vanishing of the $t \times t$ minors of the matrix $F(x)$. The singular set of $X$ is $F^{-1}\left(M_{m, n}^{t-1}\right)$, the decomposition $X=\bigcup_{i=1}^{t} X^{i}$, where $X^{i}=F^{-1}\left(M_{m, n}^{i}-M_{m, n}^{i-1}\right)$, is a stratification. The group $\operatorname{GL}_{m}\left(\mathcal{O}_{N}\right) \times \operatorname{GL}_{n}\left(\mathcal{O}_{N}\right)$, acting by conjugation, acts on the set of determinantal varieties of type $(m, n, t)$ by isomorphism.

If $F$ intersects the strata $M_{m, n}^{i}-M_{m, n}^{i-1}$ transversally at $F(x)$, then the singularity a $x$ only depends upon $\operatorname{rank}(F(x))$. We, therefore, call such a point essentially nonsingular. This naturally leads to the next definition.

Definition 2.1. Let $X$ be a determinantal singularity defined by the map $F$. Then $X$ is an essentially isolated determinantal singularity (or EIDS for short) if all points $x \in X-\{0\}$ are essentially nonsingular.

An EIDS is of course not smooth, but the singularities away from $\{0\}$ are controlled, i.e. they only depend on the strata they belong to. An example of an EIDS is any complete intersection given the type of a $(1, m, 1)($ or $(m, 1,1))$ determinantal singularity.

If $(X, 0)$ is a determinantal singularity of type $(m, n, t)$ given by $F: U \subset \mathbb{C}^{N} \rightarrow$ $M_{m, n}$ satisfying $F(0) \neq 0$, then one can find another map $F^{\prime}: U^{\prime} \subset \mathbb{C}^{N} \rightarrow$ $M_{m-s, n-s}$ with $F^{\prime}(0)=0$ such that $F^{\prime}$ gives $(X, 0)$ the structure of a determinantal singularity of type $(m-s, n-s, t-s)$ where $U$ and $U^{\prime}$ are open neighbourhoods of the origin and $s=\operatorname{rank} F(0)$. This can be done by conjugating $F$ to be on the form $\left(\begin{array}{c|c}\mathrm{id}_{s} & 0 \\ \hline 0 & F^{\prime}\end{array}\right)$ in a neighbourhood of 0 .

2.2. Transformations. As the article is about Tjurina transforms and resolutions of determinantal singularities, we will define what we mean by a transformation. 
Definition 2.2. Let $X$ be a variety and $A \subset X$ a closed subvariety, then a transformation of $(X, A)$ is a variety $\widetilde{X}$ together with a proper map $\pi: \widetilde{X} \rightarrow X$, such that $\pi: \pi^{-1}(X-A) \rightarrow X-A$ is an isomorphism and $\overline{\pi^{-1}(X-A)}=\widetilde{X}$.

The last requirement insures that $\operatorname{dim}\left(\pi^{-1}(A)\right)<\operatorname{dim}(X)$.

A resolution of $(X, \operatorname{Sing} X)$ is then just a transformation where $\widetilde{X}$ is smooth. We want to compare the different transformations, so we define a map between transformations as follows.

Definition 2.3. Let $f: T_{1} \rightarrow T_{2}$ be a map between two different transformations $\pi_{i}:\left(T_{i}, E_{i}\right) \rightarrow(X, A)$ of the same space and subspace. Then we call $f$ a map of transformations if $\pi_{1}=\pi_{2} \circ f$. We call a map of transformation an isomorphism, if it is an isomorphism of varieties.

\section{Resolutions of the Model Determinantal Singularities}

In GZĖ09] Ebeling and Gusein-Zade introduce 3 different natural ways to resolve the model determinantal singularities $M_{m, n}^{t}$. The first is the same as the Tjurina transform of $\left(M_{m, n}^{t}, M_{m, n}^{t-1}\right)$ used by Tjurina [Tju68, Van Straten vS87] and Frühbis-Krüger and Zach in [FZ15. It is defined as the following set in $M_{m, n} \times \operatorname{Gr}(n-t+1, n):$

$$
\begin{aligned}
\operatorname{Tjur}\left(M_{m, n}^{t}\right): & =\left\{(A, W) \in M_{m, n} \times \operatorname{Gr}(n-t+1, n) \mid A(W)=0\right\} \\
& =\left\{(A, W) \in M_{m, n} \times \operatorname{Gr}(n-t+1, n) \mid W \subset \operatorname{ker}(A)\right\}
\end{aligned}
$$

by considering $A \in M_{m, n}^{t}$ as a linear map $A: \mathbb{C}^{n} \rightarrow \mathbb{C}^{m}$. It is shown in ACGH85, that this is a smooth variety. Consider $\pi: \operatorname{Tjur}\left(M_{m, n}^{t}\right) \rightarrow M_{m, n}^{t}$ the restriction of the projection to the first factor. Then over the regular part of $M_{m, n}^{t}$ we have that the map $A \rightarrow(A, \operatorname{ker} A)$ is an inverse to $\pi$, hence $\pi: \operatorname{Tjur}\left(M_{m, n}^{t}\right) \rightarrow M_{m, n}^{t}$ is a resolution. Corollary 3.3 in [FZ15] shows that their definition gives the same as this one, their proof also works for general $n, m$.

The second resolution is as the Tjurina, but considering $A \in M_{m, n}^{t}$ as a linear map $A: \mathbb{C}^{m} \rightarrow \mathbb{C}^{n}$. This is of course the map given by the transpose of $A$, so we get the following:

$$
\begin{aligned}
\operatorname{Tjur}^{T}\left(M_{m, n}^{t}\right): & =\left\{(A, W) \in M_{m, n} \times \operatorname{Gr}(m-t+1, m) \mid A^{T}(W)=0\right\} \\
& =\left\{(A, W) \in M_{m, n} \times \operatorname{Gr}(m-t+1, m) \mid W \subset \operatorname{ker}\left(A^{T}\right)\right\} \\
& =\left\{(A, W) \in M_{m, n} \times \operatorname{Gr}(m-t+1, m) \mid W \subset \operatorname{Coker}(A)\right\}
\end{aligned}
$$

It is clear from the definition that this is also a smooth variety, the same proof as in the case of Tjurina transform works. If one chooses an inner product on $\mathbb{C}^{m}$, then one gets that $W \subset \operatorname{Coker}(A)$ is the same as $\operatorname{Im}(A) \subset W^{\perp}$ where $W^{\perp}$ is the orthogonal complement with respect to the inner product. The choice of inner product also gives an isomorphism between $\operatorname{Gr}(m-t+1, m)$ and $\operatorname{Gr}(t-1, m)$ defined by sending $W$ to $W^{\perp}$. Using this we get that this transform is also:

$$
\operatorname{Tjur}^{T}\left(M_{m, n}^{t}\right)=\left\{(A, W) \in M_{m, n} \times \operatorname{Gr}(t-1, m) \mid \operatorname{Im}(A) \subset W\right\} .
$$

The third resolution considered by Ebeling and Gusein-Zade is the Nash transform of $M_{m, n}^{t}$. In section one of GZĖ09 they show how to get the Nash transform which can be stated as the following proposition:

Proposition 3.1. For a model determinantal singularity the Nash transform can be given as the following:

$$
\begin{array}{r}
\operatorname{Nash}\left(M_{m, n}^{t}\right)=\left\{\left(A, W_{1}, W_{2}\right) \in M_{m, n} \times \operatorname{Gr}(n-t+1, n) \times \operatorname{Gr}(t-1, m)\right. \\
\left.\mid \operatorname{ker}(A) \supset W_{1} \text { and } \operatorname{Im}(A) \subset W_{2}\right\} .
\end{array}
$$


Proof. In ACGH85] they show that for $A \in M_{m, n}^{t}-M_{m, n}^{t-1}$, that is the regular points, that $T_{A} M_{m, n}^{t}=\left\{B \in M_{m, n} \mid B(\operatorname{ker}(A)) \subset \operatorname{Im}(A)\right\}$. Consider the map $\alpha: \operatorname{Gr}(n-t+1, n) \times \operatorname{Gr}(t-1, m) \rightarrow \operatorname{Gr}\left(d_{m, n}^{t}, m n\right)$, where $d_{m, n}^{t}:=m n-(m-t+$ 1) $(n-t+1)=\operatorname{dim}\left(M_{m, n}^{t}\right)$, given by $\alpha\left(W_{1}, W_{2}\right)=\left\{B \in M_{m, n} \mid B\left(W_{1}\right) \subset W_{2}\right\}$.

We will first show that $\alpha$ is injective. Assume that there exist two pairs $\left(W_{1}, W_{2}\right)$ and $\left(V_{1}, V_{2}\right)$ such that $\alpha\left(W_{1}, W_{2}\right)=\alpha\left(V_{1}, V_{2}\right)$. Assume that $W_{1} \neq V_{1}$, let $v_{1} \in V_{1}$ and $v_{1} \notin W_{1}$, since $\operatorname{dim}\left(W_{1}\right)=\operatorname{dim}\left(V_{1}\right)$ such $v_{1}$ exists, and choose $v_{2} \notin V_{2}$. Define the linear map $B$ as the map that sends $a v_{1}$ to $a v_{2}$ and anything else to 0 . Then $B\left(W_{1}\right)=\{0\} \subset W_{2}$ and hence $B \in \alpha\left(W_{1}, W_{2}\right)$, but $B\left(V_{1}\right)=\operatorname{Span}\left\{v_{2}\right\} \not \subset V_{2}$, so $B \notin \alpha\left(V_{1}, V_{2}\right)$ so we have a contradiction. Assume now that there exist pairs $\left(W_{1}, W_{2}\right)$ and $\left(W_{1}, V_{2}\right)$ such that $\alpha\left(W_{1}, W_{2}\right)=\alpha\left(W_{1}, V_{2}\right)$. Assume that $W_{2} \neq V_{2}$, let $v_{1} \in W_{1}$ and let $v_{2} \in V_{2}$ and $v_{2} \notin W_{2}$, since $\operatorname{dim}\left(W_{2}\right)=\operatorname{dim}\left(V_{2}\right)$ such $v_{2}$ exists. Define $B$ as the linear map that sends $a v_{1}$ to $a v_{2}$. Then $B\left(W_{2}\right)=\operatorname{Span}\left\{v_{2}\right\} \subset V_{2}$ so $B \in \alpha\left(W_{1}, V_{2}\right)$, but $\operatorname{Span}\left\{v_{2}\right\} \not \subset W_{2}$ so $B \notin \alpha\left(W_{1}, W_{2}\right)$ so we have a contradiction. This shows that $\alpha$ is injective.

Next we will show that $\alpha$ is continuous. Let $\left(V_{i}, W_{i}\right) \in \operatorname{Gr}(n-t+1, n) \times \operatorname{Gr}(t-$ $1, m)$ be a convergent sequence and let $(V, W)=\lim \left(V_{i}, W_{i}\right)$. Let $\mathcal{B}_{i}=\alpha\left(V_{i}, W_{i}\right)$, and choose a convergent subsequence $\mathcal{B}_{i}^{\prime}$ which exists because $\operatorname{Gr}\left(d_{m, n}^{t}, m n\right)$ is compact. Let $\mathcal{B}=\lim \mathcal{B}_{i}^{\prime}$, choose $B \in \mathcal{B}$ and $B_{i} \in \mathcal{B}_{i}^{\prime}$ a sequence of matrices converging to $B$. Let $v \in V$ and $v_{i} \in V_{i}$ a sequence converging to $v$, set $w_{j}=B_{j} v_{j}$ for any $j$ where $B_{j}$ is defined. Now since $B_{j}$ and $v_{j}$ converge, $w_{j}$ converges to $w=B v$, but $w_{j} \in W_{j}$ and hence its limit is in $W$. So for all $v \in V$ and all $B \in \mathcal{B}$ $B v \in W$, hence $\mathcal{B} \subset \alpha(V, W)$, but since $\operatorname{dim}(\mathcal{B})=\operatorname{dim}(\alpha(V, W))$ we have that $\mathcal{B}=\alpha(V, W)$. So any convergent subsequence of $\mathcal{B}_{i}$ converges to $\alpha(V, W)$, this implies that $\mathcal{B}_{i}$ converges to $\alpha(V, W)$ since $\operatorname{Gr}\left(d_{m, n}^{t}, m n\right)$ is compact. Therefore, $\lim \alpha\left(V_{i}, W_{i}\right)=\alpha\left(\lim \left(V_{i}, W_{i}\right)\right)$ for all convergent sequences, hence $\alpha$ is continuous.

Since $\alpha$ is a continuous map from a compact space to a compact space it is closed, and since it is injective it implies it is an embedding.

Let $\beta:\left(M_{m, n}^{t}-M_{m, n}^{t-1}\right) \rightarrow M_{m, n} \times \operatorname{Gr}(n-t+1, n) \times \operatorname{Gr}(t-1, m)$ be the map $\beta(A)=(A, \operatorname{ker}(A), \operatorname{Im}(A))$. Let $\alpha^{\prime}: M_{m, n} \times \operatorname{Gr}(n-t+1, n) \times \operatorname{Gr}(t-1, m) \rightarrow$ $M_{m, n} \times \operatorname{Gr}\left(d_{m, n}^{t}, m n\right)$ defined by $\alpha^{\prime}(A, V, W)=(A, \alpha(V, W))$. Then $\alpha^{\prime} \circ \beta(A)=$ $(A, \mathcal{B})$, where

$$
\mathcal{B}=\alpha(\operatorname{ker}(A), \operatorname{Im}(A))=\left\{B \in M_{m, n} \mid B(\operatorname{ker}(A)) \subset \operatorname{Im}(A)\right\}=T_{A} M_{m, n}^{t}
$$

So $\alpha^{\prime} \circ \beta$ is the same as the Gauss map on the regular part of $M_{m, n}^{t}$. Then we have that $\operatorname{Nash}\left(M_{m, n}^{t}\right)=\overline{\alpha^{\prime} \circ \beta\left(M_{m, n}^{t}-M_{m, n}^{t-1}\right)}$. Since $\alpha$ and hence $\alpha^{\prime}$ is a closed embedding we have $\operatorname{Nash}\left(M_{m, n}^{t}\right)=\alpha^{\prime}\left(\overline{\beta\left(M_{m, n}^{t}-M_{m, n}^{t-1}\right)}\right)$. Moreover, since $\alpha^{\prime}$ is an embedding it follows that $\operatorname{Nash}\left(M_{m, n}^{t}\right)$ is homeomorphic to $\overline{\beta\left(M_{m, n}^{t}-M_{m, n}^{t-1}\right)}$.

The last part of the proof is determining $\overline{\beta\left(M_{m, n}^{t}-M_{m, n}^{t-1}\right)}$. Now $\beta\left(M_{m, n}^{t}-\right.$ $\left.M_{m, n}^{t-1}\right)=\left\{(A, \operatorname{ker} A, \operatorname{Im} A) \in M_{m, n} \times \operatorname{Gr}(n-t+1, n) \times \operatorname{Gr}(t-1, m)\right\}$ and we want to show that the closure is $\left\{(A, V, W) \in M_{m, n} \times \operatorname{Gr}(n-t+1, n) \times \operatorname{Gr}(t-1, m) \mid \operatorname{ker}(A) \supset\right.$ $V$ and $\operatorname{Im}(A) \subset W\}=\mathcal{N}$. First assume that $(A, V, W) \in \overline{\beta\left(M_{m, n}^{t}-M_{m, n}^{t-1}\right)}$ is not in $\mathcal{N}$. This implies that that there is a $v \in V$ such that $A v \notin W$. Let $\left(A_{i}, V_{i}, W_{i}\right)$ be a sequence in $(A, V, W) \in \overline{\beta\left(M_{m, n}^{t}-M_{m, n}^{t-1}\right)}$ converging to $(A, V, W)$ and $v_{i} \in V_{i}$ a sequence converging to $v$, then $A_{i} v_{i}$ converge to $A v$ but $A_{i} v_{i}=0$ so this contradicts $A v \notin \mathcal{N}$. Let $(A, V, W) \in \mathcal{N}$ and let $r=\operatorname{rank} A$. Now $V \subset \operatorname{ker} A$, so let $V^{\prime} \subset \mathbb{C}^{n}$ be the subspace satisfying $V \oplus V^{\prime}=\operatorname{ker} A$, and $\operatorname{Im} A \subset W$ so let $W^{\prime} \subset \mathbb{C}^{m}$ be the subspace satisfying $\operatorname{Im} A \oplus W^{\prime}=W$. Let $A^{\prime}$ be a matrix of rank $t-1-r$, such that $\operatorname{ker} A^{\prime} \oplus V^{\prime}=\mathbb{C}^{n}$ and $\operatorname{Im} A^{\prime}=W^{\prime}$, such a matrix exist since $\operatorname{dim} V^{\prime}=\operatorname{dim} W^{\prime}=$ $t-1-r$. Set $A_{i}=A+\frac{1}{i} A^{\prime}$ then $\operatorname{ker} A_{i}=\operatorname{ker} A \bigcap \operatorname{ker} \frac{1}{i} A^{\prime}=\operatorname{ker} A \bigcap \operatorname{ker} A^{\prime}=V$ 
and $\operatorname{Im} A_{i}=\operatorname{Im} A+\operatorname{Im} \frac{1}{i} A^{\prime}=W$. Hence $\left(A_{i}, V_{i}, W_{i}\right):=\left(A_{i}, V, W\right)$ is a sequence in $\beta\left(M_{m, n}^{t}-M_{m, n}^{t-1}\right)$ converging to $(A, V, W)$, so $\mathcal{N} \subset \overline{\beta\left(M_{m, n}^{t}-M_{m, n}^{t-1}\right)}$ which finishes the proof.

An important consequence of this is the following:

Corollary 3.2. $\operatorname{Nash}\left(M_{m, n}^{t}\right)$ is smooth.

Proof. Using the description of $\operatorname{Nash}\left(M_{m, n}^{t}\right)$ given in Proposition 3.1 we get that the projection to the two last factors $\operatorname{Gr}(n-t+1, n) \times \operatorname{Gr}(t-1, m)$ gives $\operatorname{Nash}\left(M_{m, n}^{t}\right)$ the structure of the total space of a vector bundle over a smooth manifold.

It follows from Definition 2.3 and Proposition 3.1, that we have a map of transformations $f: \operatorname{Nash}\left(M_{m, n}^{t}\right) \rightarrow \operatorname{Tjur} M_{m, n}^{t}$ by setting $f(A, V, W)=(A, V)$ and a map of transformations $f: \operatorname{Nash}\left(M_{m, n}^{t}\right) \rightarrow \operatorname{Tjur}^{T} M_{m, n}^{t}$ by setting $f(A, V, W)=(A, W)$ and using (11). These maps are never isomorphism, as we will see later when we determine the homotopy type of these spaces. Now finding maps between Tjur $M_{m, n}^{t}$ and $\operatorname{Tjur}^{T} M_{m, n}^{t}$ turns out to be impossible by the following result:

Proposition 3.3. There exist no continuous map of transformations between Tjur $M_{m, n}^{t}$ and $\operatorname{Tjur}^{T} M_{m, n}^{t}$.

Proof. We start by using (11) to identify $\operatorname{Tjur}^{T}\left(M_{m, n}^{t}\right)$ with $\left\{(A, W) \in M_{m, n} \times\right.$ $\operatorname{Gr}(t-1, m) \mid \operatorname{Im}(A) \subset W\}$. Let $f: \operatorname{Tjur}\left(M_{m, n}^{t}\right) \rightarrow \operatorname{Tjur}^{T} M_{m, n}^{t}$ be a map of transformations, this implies that over $\pi^{-1}\left(M_{m, n}^{t}-M_{m, n}^{t-1}\right)$ we have that $f(A, \operatorname{ker} A)=$ $(A, \operatorname{Im} A)$. Let $\left\{x_{1}, \ldots, x_{n}\right\}$ be a basis of $\mathbb{C}^{n}$ and $\left\{y_{1}, \ldots, y_{m}\right\}$ be a basis for $\mathbb{C}^{m}$. Let $A$ be the matrix in this basis of the linear map $A\left(x_{1}, \ldots, x_{n}\right)=\left(x_{1}, \ldots, x_{t-2}, 0, \ldots, 0\right)$, notice that there is at least 2 zeros at the end since $t \leq m$. $\operatorname{rank} A=t-2$ hence $A \in M_{m, n}^{t-1}$. Let $V=\operatorname{Span}\left\{x_{t}, \ldots, x_{n}\right\}$ then it is clear that $\operatorname{ker} A \supset V$.

We now define two different sequences of matrices $A_{s}^{1}$ and $A_{s}^{2} . A_{s}^{1}\left(x_{1}, \ldots, x_{n}\right):=$ $\left(x_{1}, \ldots, x_{t-2}, \frac{1}{s} x_{t-1}, 0,0, \ldots, 0\right)$ and $A_{s}^{2}\left(x_{1}, \ldots, x_{n}\right):=\left(x_{1}, \ldots, x_{t-2}, 0, \frac{1}{s} x_{t-1}, 0, \ldots, 0\right)$. It is clear that $\operatorname{ker} A_{s}^{i}=V$ and $\lim _{s \rightarrow \infty}\left(A_{s}^{i}, V\right)=(A, V)$ for $i=1,2$. Since $A_{s}^{i} \in$ $M_{m, n}^{t}-M_{m, n}^{t-1}$ we get that $f\left(A_{s}^{i}, V\right)=\left(A_{s}^{i}, \operatorname{Im} A_{s}^{i}\right)$. Now let $W_{1}:=\operatorname{Span}\left\{y_{1}, \ldots, y_{t-1}\right\}=$ $\operatorname{Im} A_{s}^{1}$ and $W_{2}:=\operatorname{Span}\left\{y_{1}, \ldots, y_{t-2}, y_{t}\right\}=\operatorname{Im} A_{s}^{2}$. If $f$ is continuous, then we have that $f(A, W)=f\left(\lim _{s \rightarrow \infty}\left(A_{s}^{i}, V\right)\right)=\lim _{s \rightarrow \infty} f\left(A_{s}^{i}, V\right)=\left(A, W_{i}\right)$ for $i=1,2$. But $W_{1} \neq W_{2}$ hence $f$ can not be continuous. The argument that there is no continuous map of transformations from $\operatorname{Tjur}^{T} M_{m, n}^{t}$ to Tjur $M_{m, n}^{t}$ is similar.

Next we determine the homotopy type of the transformations, and the above shows that even in the case Tjur $M_{m, n}^{t}$ and $\operatorname{Tjur}^{T} M_{m, n}^{t}$ are homotopy equivalent they are not isomorphic.

Proposition 3.4. Let $\pi:\left(T\left(M_{m, n}^{t}\right), E\right) \rightarrow\left(M_{m, n}^{t}, M_{m, n}^{t-1}\right)$ be one of the 3 transforms discussed above. Then $T\left(M_{m, n}^{t}\right)$ is homotopy equivalent to $\pi^{-1}(0)$.

This gives that $\operatorname{Nash}\left(M_{m, n}^{t}\right) \sim \operatorname{Gr}(n-t+1, n) \times \operatorname{Gr}(t-1, m), \operatorname{Tjur}\left(M_{m, n}^{t}\right) \sim$ $\operatorname{Gr}(n-t+1, n)$ and $\operatorname{Tjur}^{T}\left(M_{m, n}^{t}\right) \sim \operatorname{Gr}(t+1, m)$, where $\sim$ denotes homotopy equivalence.

Proof. We will only show this for $\operatorname{Nash}\left(M_{m, n}^{t}\right)$ but the other proofs are similar. Let $F: \operatorname{Nash}\left(M_{m, n}^{t}\right) \times \mathbb{C} \rightarrow \operatorname{Nash}\left(M_{m, n}^{t}\right)$ be the map defined by $F(A, V, W, s)=$ $f_{s}(A, V, W)=(s A, V, W)$, when we use the identification for the Nash transformation given by Proposition 3.1. The map is well defined since $(s A)(V)=s(A(V))=0$ and $\operatorname{Im} s A=\operatorname{Im} A \subset W$ if $s \neq 0$ and $\operatorname{Im} s A=\{0\} \subset W$ if $s=0$. It is continuous since it is just scalar multiplication. Restrict the map to $s \in[0,1]$. Then $f_{1}=\mathrm{id}$, 
$\left.f_{s}\right|_{\pi^{-1}(0)}=\left.i d\right|_{\pi^{-1}(0)}$ and $f_{0}\left(\operatorname{Nash}\left(M_{m, n}^{t}\right)\right)=\pi^{-1}(0)$. Hence $f_{s}$ is a deformation retraction, and $\operatorname{Nash}\left(M_{m, n}^{t}\right)$ is homotopy equivalent to $\pi^{-1}(0)$.

\section{Transformations of GEnERAL DEterminantal Singularities}

In this section we will introduce the transformations defined above for general determinantal varieties. We start by introducing the Tjurina transform. The Tjurina transform of determinantal singularities has be introduced several places before for example [Tju68], vS87], [ACGH85], GZĖ09] and [FZ15]. They in general define the Tjurina transform of a determinantal singularity $X$ of type $(m, n, t)$ given by $F: \mathbb{C}^{n} \rightarrow M_{m, n}$ as the fibre product $X \times_{F} \operatorname{Tjur}\left(M_{m, n}^{t}\right)$, which works very well in the cases they consider. But this definition gives the following problem in a more general setting: Assume that $\operatorname{dim}(X) \leq(t-1)(n-t+1)$ and let $p: X \times_{F} \operatorname{Tjur}\left(M_{m, n}^{t}\right) \rightarrow X$ be the projection to the first factor. Then $p^{-1}(0) \cong \operatorname{Gr}(n-t+1, n)$, hence the exceptional fibre of $p$ has dimension greater than or equal to the dimension of $X$. This means that the fibre product does not satisfy the conditions to be a transformation given in Definition 2.2. We will instead give an alternative definition that does not have this problem. It should be said that in GZĖ09] and [FZ15] they only consider the Tjurina transformation in situations where this does not happen, and that our definition agrees with theirs in these cases. We will in Proposition 4.4 see when the two definitions agree in general.

Definition 4.1. Let $X$ be a determinantal singularity of type $(m, n, t)$ given by $F: \mathbb{C}^{n} \rightarrow M_{m, n}$, define $B: X_{\text {reg }} \rightarrow \operatorname{Gr}(t-1, n)$ as the map that sends $x$ into the row space of $F(x)$. Then we define the Tjurina transform $\operatorname{Tjur}(X)$ of $X$ as

$$
\operatorname{Tjur}(X):=\overline{\left\{(x, W) \in X_{\text {reg }} \times \operatorname{Gr}(t-1, n) \mid W=B(x)\right\}} \subset X \times \operatorname{Gr}(t-1, n),
$$

and we define the map $\pi^{T j}: \operatorname{Tjur}(X) \rightarrow X$ as the projection to the first factor.

It is clear that this satisfies the assumptions of Definition 2.2 to be a transformation of $\left(X, X_{\text {sing }}\right)$ since $\left.\pi^{T j}\right|_{\operatorname{Tjur}(X)-\pi_{T j}^{-1}\left(X_{\text {sing }}\right)}$ is the inverse of $B$, it is proper since all fibres are either points or closed subsets of $\operatorname{Gr}(t-1, n)$ hence compact, and $\operatorname{dim}\left(\left(\pi^{T j}\right)^{-1}\left(X_{\text {sing }}\right)\right)<\operatorname{dim}(X)$ since it is a closed subvariety of an algebraic variety of $\operatorname{dim}(X)$.

Notice that the choice of an inner product on $\mathbb{C}^{n}$ gives a one to one correspondence between the row space of $F(x)$ and $\operatorname{ker} F(x)$ and an isomorphism $\operatorname{Gr}(t-1, n) \cong \operatorname{Gr}(n-t+1, n)$. Hence we get that

$$
\operatorname{Tjur}(X)=\overline{\left\{(x, W) \in X_{\text {reg }} \times \operatorname{Gr}(n-t+1, n) \mid W=\operatorname{ker} F(x)\right\}} .
$$

We use the row space in our definition, since it makes calculation easier as we see later.

Proposition 4.2. Let $X$ be a complete intersection and not a hypersurface of type $(m, m, m)$, then $\operatorname{Tjur}(X)=X$.

Proof. A complete intersection is a determinantal singularity of type $(m, n, 1)$ if it is not a hypersurface of type $(m, m, m)$. Hence $\operatorname{Tjur}(X) \subset X \times \operatorname{Gr}(0, n)=X$ and $B$ is constant. The result then follows since $\operatorname{Tjur}(X)=\overline{X_{\text {reg }}}$ and the regular points is an open dense subset of $X$.

We will later see some examples of hypersurfaces of type $(m, m, m)$ where the Tjurina transform is useful in simplifying singularities. 
To study the local properties of the Tjurina transform closer we will use the following matrix charts on $\operatorname{Gr}(t-1, n)$. Let $I \subset\{1, \ldots, n\}$ such that $\# I=t-1$. For each such $I=\left\{i_{1}, \ldots, i_{t-1}\right\}$ we define a chart of $\operatorname{Gr}(t-1, n)$ by the $(t-1) \times n$ matrix $A_{I}$ which consists of the columns $C_{i}$ given as follows:

$$
C_{i}=\left(\begin{array}{c}
a_{1 i} \\
\vdots \\
a_{(t-1) i}
\end{array}\right) \text { if } i \notin I, \text { and } C_{i_{l}}=\left(\begin{array}{c}
0 \\
\vdots \\
0 \\
1 \\
0 \\
\vdots \\
0
\end{array}\right) \text { the } l^{t h} \text { entry. }
$$

Here we consider $a_{j i} \in \mathbb{C}$ as variables. Let $a=\left(a_{1 i_{1}}, \ldots, a_{(t-1) i_{n-t+1}}\right) \in \mathbb{C}^{(t-1)(n-t+1)}$, hence $A_{I}(a)$ define a map $\tilde{A}_{I}: \mathbb{C}^{(t-1)(n-t+1)} \rightarrow \operatorname{Gr}(t-1, n)$ by sending $a$ to the row space of $A_{I}(a) . \quad\left\{\tilde{A}_{I}\right\}_{I}$ is a cover of $\operatorname{Gr}(t-1, n)$ by algebraic maps, and if $U_{I}=\operatorname{Im} \tilde{A}_{I}$ the change of coordinates from $\tilde{A}_{I}^{-1}\left(U_{I} \cap U_{J}\right)$ to $\tilde{A}_{J}^{-1}\left(U_{I} \cap U_{J}\right)$ is given by $A_{J}^{T} A_{I}(a)$.

To see the row space of $F(x)$ in a given chart $A_{I}$, we construct the following $(m+t-1) \times n$ matrix:

$$
\tilde{F}_{I}^{T j}(x, a)=\left(\frac{A_{I}(a)}{F(x)}\right) .
$$

Then the row space of $F(x)$ is contained in $\tilde{A}_{I}(a)$ if and only if $\operatorname{rank} \tilde{F}_{I}^{T j}(x, a)=t-1$.

Let $\widetilde{\operatorname{Tjur}}_{I}(X)=\left(\tilde{F}_{I}^{T j}\right)^{-1}\left(M_{m+t-1, n}^{t}\right) \subset X \times \mathbb{C}^{(t-1)(n-t+1)}$, and $\tilde{\pi}_{I}^{T j}: \widetilde{\operatorname{Tjur}}_{I}(X) \rightarrow$ $X$ be the projection to the first factor.

It is clear from the above construction that $\operatorname{Tjur}_{I}(X):=\operatorname{Tjur}(X) \bigcap \operatorname{Im} \tilde{A}_{I} \subset$ $\widetilde{\operatorname{Tjur}}_{I}(X)$ but they are not necessarily equal, moreover, $\widetilde{\operatorname{Tjur}}{ }_{I}(X)$ is not necessarily a determinantal singularity. We have that $\left(\pi_{I}^{T j}\right)^{-1}\left(X_{r e g}\right)=\left(\tilde{\pi}_{I}^{T j}\right)^{-1}\left(X_{r e g}\right)$ this implies that $\operatorname{dim} \widetilde{\operatorname{Tjur}}_{I}(X)=\max \left(\operatorname{dim} X, \operatorname{dim}\left(\tilde{F}_{I}^{T j}\right)^{-1}\left(M_{m+t-1, n}^{t-1}\right)\right)$. Now $\operatorname{dim}\left(\tilde{F}_{I}^{T j}\right)^{-1}\left(M_{m+t-1, n}^{t-1}\right)$ is the largest of the dimensions of the pullback of the strata of $M_{m+t-1, n}^{t-1}$. So $\left(\tilde{F}_{I}^{T j}\right)^{-1}\left(M_{m+t-1, n}^{s}-M_{m+t-1, n}^{s-1}\right) \subset X \times \operatorname{Gr}(t-1, n)$ consist of the pairs $(x, W)$ such that $x \in F^{-1}\left(M_{m, n}^{s}-M_{m+t-1, n}^{s-1}\right)$ and $\{$ row space of $F(x)\} \subset W$. Since $\operatorname{rank} F(x)=s-1$ we can write all such $W$ as $W=\{$ row space of $F(x)\}+$ $W_{F(x)}$ where $W_{F(x)}$ is a $t-1-s$ dimensional subspace of the complement of \{row space of $F(x)\} \subset \mathbb{C}^{n}$. Moreover, we have that for any $V$ in the complement of $\{$ row space of $F(x)\} \subset \mathbb{C}^{n}$ we have that $\operatorname{rank}\{$ row space of $F(x)\}+V=t-1$. Hence we get that $\{W \in \operatorname{Gr}(t-1, n) \mid$ row space of $F(x)\} \subset W\}$ is isomorphic to $\operatorname{Gr}(t-1-s, n-s)$. So we get that $\left.\operatorname{dim}\left(\tilde{F}_{I}^{T j}\right)^{-1}\left(M_{m+t-1, n}^{s}-M_{m+t-1, n}^{s-1}\right)\right)=$ $\operatorname{dim} F^{-1}\left(\left(M_{m+t-1, n}^{s}-M_{m+t-1, n}^{s-1}\right)+\operatorname{dim} \operatorname{Gr}(t-1-s, n-s)\right.$.

The above implies that $\operatorname{dim} \widetilde{\operatorname{Tjur}}_{I}(X)=\operatorname{dim} \operatorname{Tjur}_{I}(x)=\operatorname{dim} X$ if and only if $\operatorname{dim} F^{-1}\left(\left(M_{m+t-1, n}^{s}-M_{m+t-1, n}^{s-1}\right) \leq \operatorname{dim} X-\operatorname{dim} \operatorname{Gr}(t-1-s, n-s)=N-(m-\right.$ $t+1)(n-t+1)-(t-s)(n-t+1)=N-(m-s+1)(n-t+1)$ for all $s=1, \ldots, t$. If $X$ has an isolated singularity, this becomes $N \geq m(n-t+1)$.

Proposition 4.3. If $\operatorname{dim} \widetilde{\operatorname{Tjur}}_{I}(X)=\operatorname{dim} X$ then $\widetilde{\operatorname{Tjur}}_{I}(X)$ is a determinantal singularity.

Proof. We just need to check if codim $\widetilde{\operatorname{Tjur}}_{I}(X)=\operatorname{codim} M_{m+t-1, n}^{t}=(m+t-$ $1-t+1)(n-t+1)=m(n-t+1)$. But codim $\widetilde{\operatorname{Tjur}}_{I}(X)=\operatorname{codim} \operatorname{Tjur}_{I}(X)=$ $\operatorname{codim} X+(t-1)(n-t+1)=(m+t-1)(n+t-1)+(t-1)(n+t-1)=m(n-t+1)$. 
In this case we get that $\widetilde{\operatorname{Tjur}}_{I}(X)$ is a determinantal singularity of type $(m+$ $t-1, n, t)$. But $\operatorname{rank} \tilde{F}_{I}^{T j}(0,0)=t-1$, so one can find another matrix $F_{I}^{\prime}(x, a)$ defining $\widetilde{\operatorname{Tjur}}_{I}(X)$ such $F_{I}^{\prime}(0,0)=0$ and this is a determinantal singularity of type $(m+t-1-(t-1), n+(t-1), t-(t-1))=(m, n+t-1,1)$. Since codim $\widetilde{\operatorname{Tjur}}_{I}(X)=$ $m(n-t+1)$ we get that $\widetilde{\operatorname{Tjur}}_{I}(X)$ is a complete intersection. We will later show how to explicitly find $F_{I}^{\prime}(x, a)$ also in the case $\operatorname{dim} \widetilde{\operatorname{Tjur}}_{I}(X) \neq \operatorname{dim} X$.

We can also use this to determine when $\operatorname{Tjur}_{I}(X)$ and $\widetilde{\operatorname{Tjur}}_{I}(X)$ are equal. Notice that $\widetilde{\operatorname{Tjur}_{I}}(X)=\left(X \times_{F} \operatorname{Tjur}\left(M_{m, n}^{t}\right)\right) \cap \operatorname{Im} \tilde{A}_{I}$, hence the next proposition also answers the question, when is our definition of Tjurina transform the same as the one used by other authors.

Proposition 4.4. $\widetilde{\operatorname{Tjur}}_{I}(X)=\operatorname{Tjur}_{I}(X)$ if and only if $\operatorname{dim} X^{s}<N-(m-s+$ 1) $(n-t+1)$ for all $s \in 1, \ldots, t-1$.

Proof. Since $\operatorname{Tjur}(X)$ is a transformation, we have that $\operatorname{dim} \pi^{T j}\left(X^{t-1}\right)<\operatorname{dim} X$, remember the $X^{t-1}$ is the singular set of $X$. Then the above calculations of the dimensions of the fibres give the inequalities, and we get the only if direction.

So assume that the inequalities are satisfied, this implies that $\operatorname{dim} \operatorname{Tjur}_{I}(X)=$ $\operatorname{dim} \widetilde{\operatorname{Tjur}}_{I}(X)$ and that $\operatorname{dim}\left(\tilde{\pi}_{I}^{T j}\right)^{-1}\left(X^{t-1}\right)<\operatorname{dim} X$. Now $\operatorname{Tjur}_{I}(X)$ is an irreducible component of $\widetilde{\operatorname{Tjur}}_{I}(X)$, and $\operatorname{Tjur}_{I}(X)$ is not a proper subvariety of any irreducible variety of the same dimension, since it is closed. This implies that if $\widetilde{\operatorname{Tjur}}_{I}(X) \neq \operatorname{Tjur}_{I}(X)$ then there exist an other irreducible component $V \subseteq$ $\widetilde{\operatorname{Tjur}}_{I}(X)$ different from $\operatorname{Tjur}_{I}(X)$. But since $\widetilde{\operatorname{Tjur}}_{I}(X)$ is a complete intersection it is equidimensional, and hence $\operatorname{dim} V=\operatorname{dim} \operatorname{Tjur}_{I}(X)$. Since $\left(\pi_{I}^{T j}\right)^{-1}\left(X_{\text {reg }}\right)=$ $\left(\tilde{\pi}_{I}^{T j}\right)^{-1}\left(X_{\text {reg }}\right)$ we have that $V \subset\left(\tilde{\pi}_{I}^{T j}\right)^{-1}\left(X^{t-1}\right)$, but this is a contradiction since $\operatorname{dim} V>\operatorname{dim} \tilde{\pi}_{I}^{T j}\left(X^{t-1}\right)$.

We now want to give an explicit method to find $F_{I}^{\prime}(x, a)$. Assume that $I=$ $i_{1}, \ldots, i_{t-1} \subset\{1, \ldots, n\}$ as before. Now by adding columns of the form $-a_{i j} C_{i_{j}}$ to the $i$ 'th column, for all $i \notin I$ and all $j=1, \ldots, t-1$, we get a matrix which has $t-1$ linearly independent rows $R_{i_{j}}$ of the form $R_{i_{j}}=(0, \ldots, 0,1,0, \ldots, 0)$, where the 1 is the $i_{j}$ entry. To this matrix we then add rows of the form $-f_{i i_{j}}(x) R_{i_{j}}$ to the $i$ 'th row for $i=t, \ldots, m$ and $j=1, \ldots, t-1$. We now have a matrix $\bar{F}_{I}(x, a)$ consisting of the following columns:

$$
\bar{F}_{i}=\left(\begin{array}{c}
0 \\
\vdots \\
0 \\
f_{1 i}(x)-\sum_{j=1}^{t-1} a_{j i} f_{1 i_{j}}(x) \\
\vdots \\
f_{m i}(x)-\sum_{j=1}^{t-1} a_{j i} f_{m i_{j}}(x)
\end{array}\right) \text { if } i \notin I, \text { and } \bar{F}_{i_{l}}=\left(\begin{array}{c}
0 \\
\vdots \\
0 \\
1 \\
0 \\
\vdots \\
0
\end{array}\right) \text { the } l^{t h} \text { entry. }
$$

The $t \times t$ minors of $\bar{F}_{I}(x, a)$ still defines $\widetilde{\operatorname{Tjur}}_{I}(X)$. Notice that we can choose of special minors $\Delta_{i, j}$, where $i \in\{1, \ldots, m\}$ and $j \notin I$, where each row and each column have a single non zero entry, which is 1 except for the $i i_{j}$ entry which is $f_{i j}(x)-\sum_{j=1}^{t-1} a_{j i} f_{i i_{j}}(x)$. This implies that $\widetilde{\operatorname{Tjur}}_{I}(X)$ is defined by the $(n-t+1) m$ equations $f_{i j}(x)-\sum_{j=1}^{t-1} a_{j i} f_{i i_{j}}(x)=0$. Hence it is defined by the $1 \times 1$ minors of 
the matrix $m \times(n-t+1)$ matrix $F_{I}^{\prime}(x, a)$ with columns:

$$
F_{i}^{\prime}=\left(\begin{array}{c}
f_{1 i}(x)-\sum_{j=1}^{t-1} a_{j i} f_{1 i_{j}}(x) \\
\vdots \\
f_{m i}(x)-\sum_{j=1}^{t-1} a_{j i} f_{m i_{j}}(x)
\end{array}\right) \text { if } i \notin I .
$$

This does still not imply that $\widetilde{\operatorname{Tjur}}_{I}(X)$ is a determinantal singularity, since the codimension might not be right. Even if $\widetilde{\operatorname{Tjur}}_{I}(X)$ is a determinantal variety, it is often not irreducible, and hence $\widetilde{\operatorname{Tjur}}_{I}(X) \neq \operatorname{Tjur}_{I}(X)$, as we will see in the next examples.

Example 4.5. Let $X$ be the determinantal singularity of type $(2,3,2)$ defined by the following matrix

$$
F_{1}(x, y, z, w)=\left(\begin{array}{ccc}
w^{l} & y & x \\
z & w & y^{k}
\end{array}\right)
$$

For $k, l>2$. In this case $\widetilde{\operatorname{Tjur}_{I}}(X)$ is a determinantal variety for all $I$. $\widetilde{\operatorname{Tjur}}_{I}(X) \neq$ $\operatorname{Tjur}_{I}(X)$ in the chart defined by $I=\{2\}$, in the other charts both $\widetilde{\operatorname{Tjur}}_{I}(X)$ and $\operatorname{Tjur}_{I}(X)$ are smooth. Now lets look closer on the equations in the chart defined by $I=\{2\}$.

$$
F_{\{2\}}^{\prime}\left(x, y, z, w, a_{1}, a_{3}\right)=\left(\begin{array}{cc}
w^{l}-a_{1} y & x-a_{3} y \\
z-a_{1} w & y^{k}-a_{3} w
\end{array}\right) .
$$

Notice that the equations $x-a_{3} y=0$ and $z-a_{1} w=0$ just define $x$ and $z$ as holomorphic functions of the other variables, and give embeddings of a $\mathbb{C}^{4}$ into $\mathbb{C}^{6}$. Now if we multiply the equations $y^{k}-a_{3} w=0$ and $w^{l}-a_{1} y=0$ we get:

$$
\begin{aligned}
0 & =\left(y^{k}-a_{3} w\right)\left(w^{l}-a_{1} y\right)=y^{k} w^{l}-a_{1} y^{k+1}-a_{3} w^{l+1}+a_{1} a_{3} y w \\
& =y^{k} w^{l}-a_{1} a_{3} y w-a_{1} a_{3} y w+a_{1} a_{3} y w=y w\left(y^{k-1} w^{w-1}-a_{1} a_{3}\right) .
\end{aligned}
$$

Hence we see that $\widetilde{\operatorname{Tjur}_{I}}(X)$ is not irreducible. $y=0$ and $w=0$ both define the fibre $\left(\tilde{\pi}_{I}^{T j}\right)^{-1}(0)$ which is two dimensional and therefore can not be a subset of $\operatorname{Tjur}_{I}(X)$. Therefore, $\operatorname{Tjur}_{I}(X)$ is given by the equations $y^{k-1} w^{w-1}-a_{1} a_{3}=0$, $w^{l}-a_{1} y=0$ and $y^{k}-a_{3} w=0$. Hence it is a determinantal singularity of the same type as $X$ given by the matrix.

$$
\left(\begin{array}{ccc}
w^{l-1} & y & a_{3} \\
a_{1} & w & y^{k-1}
\end{array}\right)
$$

Example 4.6. Let $X \subset \mathbb{C}^{4}$ be a determinantal singularity of type $(3,2,2)$ given by

$$
F_{2}(x, y, z, w)=\left(\begin{array}{cc}
w^{l} & z \\
y & w \\
x & y^{k}
\end{array}\right)
$$

For $k, l>2$. The $\widetilde{\operatorname{Tjur}}_{I}(X)$ is given in the two charts $I=\{1\},\{2\}$ by the matrices

$$
F_{\{1\}}^{\prime}\left(x, y, z, w, a_{1}\right)=\left(\begin{array}{c}
z-a_{1} w^{l} \\
w-a_{1} y \\
y^{k}-a_{1} x
\end{array}\right) \text { and } F_{\{2\}}^{\prime}\left(x, y, z, w, a_{2}\right)=\left(\begin{array}{c}
w^{l}-a_{2} z \\
y-a_{2} w \\
x-a_{2} y^{k}
\end{array}\right)
$$

In this case we see that $\widetilde{\operatorname{Tjur}}_{I}(X)=\operatorname{Tjur}_{I}(X)$, and hence the Tjurina transform of $X$ is a complete intersection. 
Notice that the singularities in Example 4.5 and 4.6 are the same, it is just their representations as determinantal singularities that are different. In fact the difference is that $F_{1}(x, y, z, w)=F_{2}(x, y, z, w)^{T}$.

Let us define $\operatorname{Tjur}^{T}(X)$.

Definition 4.7. Let $X$ be a determinantal singularity of type $(m, n, t)$ given by $F: \mathbb{C}^{N} \rightarrow M_{m, n}$, define $C: X_{\text {reg }} \rightarrow \operatorname{Gr}(t-1, m)$ as the map that sends $x$ into the column space of $F(x)$. Then we define $\operatorname{Tjur}^{T}(X)$ of $X$ as

$$
\operatorname{Tjur}^{T}(X)=\overline{\left\{(x, W) \in X_{\text {reg }} \times \operatorname{Gr}(t-1, n) \mid W=C(x)\right\}} \subset X \times \operatorname{Gr}(t-1, m),
$$

and we define the map $\pi^{T j^{T}}: \operatorname{Tjur}^{T}(X) \rightarrow X$ as the projection to the first factor.

This definition gives us that $\operatorname{Tjur}^{T}(X)=\operatorname{Tjur}\left(X^{T}\right)$, where $X^{T}$ is $X$ but defined as a determinantal singularity by $F(x)^{T}: \mathbb{C}^{N} \rightarrow M_{n, m}$. This means that we can define $\widetilde{\operatorname{Tjur}_{I}^{T}}(X)$ as for $\operatorname{Tjur}(X)$, either by setting $\widetilde{\operatorname{Tjur}}_{I}^{T}(X)=\widetilde{\operatorname{Tjur}}_{I}\left(X^{T}\right)$ or by defining it using $\bar{F}_{I}^{T}(x, a):=\left(F(x) \mid A_{I}^{T}\right)$, where $I$ now is a subset of $1, \ldots, m$.

This of course immediately gives us the following results.

Proposition 4.8. $\widetilde{\operatorname{Tjur}}_{I}^{T}(X)$ is a determinantal singularity if and only if $\operatorname{dim} X^{s} \leq$ $N-(m-t+1)(n-s+1)$ for all $s \in 1, \ldots, t$.

Proposition 4.9. $\widetilde{\operatorname{Tjur}}_{I}^{T}(X)=\operatorname{Tjur}_{I}^{T}(X)$ if and only if $\operatorname{dim} X^{s}<N-(m-t+$ 1) $(n-s+1)$ for all $s \in 1, \ldots, t-1$.

Notice that this definition of $\operatorname{Tjur}^{T}\left(M_{m, n}^{t}\right)$ is the same as the one we gave earlier, since the column space of a matrix is the same as its image.

The next example shows that just like the blow-up and the Nash transform, the Tjurina transform of a normal singularity need not be normal and that the dimension of the singular set can increase under the Tjurina transform.

Example 4.10 (Tjur $(X)$ need not be normal). Let $X$ be the hypersurface singularity given by $z^{2}-x^{4}-x^{2} y^{3}-x^{2} y^{5}-y^{8}=0$ it can be given as a determinantal singularity of type $(2,2,2)$ by the matrix $\left(\begin{array}{c}z \\ x^{2}+y^{5} \\ x^{2}+y^{3} \\ z\end{array}\right)$. We get that the Tjurina transform is

$$
F_{\{1\}}^{\prime}\left(x, y, z, a_{2}\right)=\left(\begin{array}{c}
x^{2}+y^{3}-a_{2} z \\
z-a_{2}\left(x^{2}+y^{5}\right)
\end{array}\right) \text { and } F_{\{2\}}^{\prime}\left(x, y, z, a_{1}\right)=\left(\begin{array}{c}
z-a_{1}\left(x^{2}+y^{3}\right) \\
x^{2}+y^{5}-a_{1} z
\end{array}\right)
$$

In the first chart we can by change of coordinates, see that we have the hypersurface singularity $x^{2}+y^{3}-a_{2}^{2}\left(x^{2}+y^{5}\right)=0$, which has all of the $a_{2}$-axis as its singular set. In the same way the second chart gives us the hypersurface $x^{2}+y^{5}-a_{1}^{2}\left(x^{2}+y^{3}\right)=$ 0 , which has the $a_{1}$-axis as its singular set. Hence $\operatorname{Tjur}(x)$ have singularities of codimension 1 , and is, therefore, not normal. It also illustrates that the singular set of $\operatorname{Tjur}(X)$ might have larger dimension than the singular set of $X$.

We saw in Section 3 that for the model determinantal $\operatorname{singularities} \operatorname{Nash}\left(M_{m, n}^{t}\right) \cong$ $\operatorname{Tjur}\left(M_{m, n}^{t}\right) \times_{\left(M_{m, n}^{t}-M_{m, n}^{t-1}\right)} \operatorname{Tjur}^{T}\left(M_{m, n}^{t}\right)$. Is this then true in general? $\operatorname{Is} \operatorname{Nash}(X) \cong$ $\operatorname{Tjur}(X) \times_{X_{\text {reg }}} \operatorname{Tjur}^{T}(X)$ ? The answer is unfortunately no as we can see in the following. Let $X$ be the determinantal singularity defined in Example 4.5. There we saw that the exceptional divisor of $\operatorname{Tjur}(X)$ consist of two irreducible components. In Example 4.6 we got that the exceptional divisor of $\operatorname{Tjur}^{T}(X)$ is a single irreducible curve. Hence the exceptional divisor of $\operatorname{Tjur}(X) \times_{X_{\text {reg }}} \operatorname{Tjur}^{T}(X)$ consists of three irreducible curves. But in Tju68 Tjurina shows that $X$ is a minimal surface 
singularity with the following dual resolution graph.

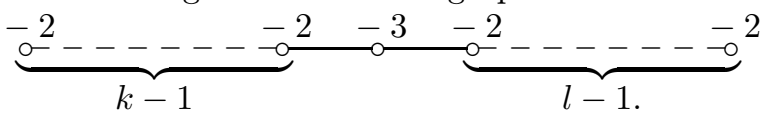

Following the work of Spivakovsky Spi90] the irreducible components of the exceptional divisor of the normalized Nash transform of a surface singularity corresponds to the irreducible components of the exceptional divisor intersecting the strict transform of the polar curve of a generic plane projection. By Theorem 5.4 in Chapter III of Spi90 we find that the polar of a generic plane projection of $X$ intersects the exceptional divisor in two different components. This implies that the exceptional divisor of $\operatorname{Nash}(X)$ has at most two components, since the number of components can not decrease under normalization. Hence $\operatorname{Nash}(X)$ and $\operatorname{Tjur}(X) \times_{X_{\text {reg }}} \operatorname{Tjur}^{T}(X)$ have non isomorphic exceptional divisors, and can, therefore, not be isomorphic as transformations.

\section{When is the TuURina transform a Complete Intersection}

In Lemma 3.14 of their articel [FZ15] Frühbis-Krüger and Zach find conditions under which the Tjurina transform of Cohen-Macauley codimension 2 singularities in $\mathbb{C}^{5}$ only has isolated singularities. They also notice in Remark 3.16 that in all the cases of simple isolated Cohen-Macauley codimension 2 singularities they consider, the Tjurina transforms are isolated local complete intersection. In this section we consider the second question and give some general condition on when the Tjurina transform of an EIDS is a local complete intersection.

If $X$ is an EIDS, remember that means that $F$ is transverse to all strata of $M_{m, n}^{t}$ in a punctured neighbourhood of the origin, then we can get the following result concerning the Tjurina transform.

Proposition 5.1. Let $X \subset \mathbb{C}^{N}$ be an EIDS of type $(m, n, t)$, then $\operatorname{Tjur}(X)$ is a local complete intersection if $N-m(n-t+1)>\operatorname{dim} X^{1}$ and $\operatorname{Tjur}^{T}(X)$ is a local complete intersection if $N-n(m-t+1)>\operatorname{dim} X^{1}$.

Proof. To show that $\operatorname{Tjur}(X)$ is a local complete intersection, it is enough to show that $\operatorname{Tjur}_{I}(X)$ is a complete intersection for all $I$. To do this we show that $\operatorname{Tjur}_{I}(X)=\widetilde{\operatorname{Tjur}}_{I}(X)$. First notice that being an EIDS implies that $\operatorname{dim} X^{s}=$ $N-(m-s+1)(n-s+1)<N-(m-s+1)(n-t+1)$ for all $s \in 2, \ldots t-1$. So for $X$ to satisfy Proposition 4.4 we just need that $\operatorname{dim} X^{1}<N-m(n-t+1)$ which follows from the assumption. So $\operatorname{Tjur}_{I}(X)=\widetilde{\operatorname{Tjur}_{I}}(X)$ and $\widetilde{\operatorname{Tjur}}_{I}(X)$ is a complete intersection. Hence $\operatorname{Tjur}(X)$ is a local complete intersection.

The proof for $\operatorname{Tjur}^{T}(X)$ is similar, just exchange $n$ and $m$.

The assumption on $N$ can replaced by assumption on $t$ and the strata of $X$ as seen in the next theorem.

Theorem 5.2. Let $X$ be an EIDS of type $(m, n, t)$, where $t \geq 3$ and $X^{2} \neq \emptyset$. Then at least one of $\operatorname{Tjur}(X)$ and $\operatorname{Tjur}^{T}(X)$ is a local complete intersection.

Proof. First notice that since $t \geq 3$ one of the following two inequalities holds $n-1<m(t-2)$ or $m-1<n(t-2)$. We will first show that if the first equation holds, then $\operatorname{Tjur}(X)$ is a local complete intersection.

Assume that $n-1<m(t-2)$. To show that that $\operatorname{Tjur}(X)$ is a complete intersection, we just need to show that $\operatorname{dim} X^{1}<N-m(n-t+1)$ by Proposition 5.1. Now $\operatorname{dim} X^{1}<\operatorname{dim} X^{2}=N-(m-1)(n-1)=N-m n+m+n-1<$ $N-m n+m+m(t-2)=N-m(n-t+1)$. So Tjur $(X)$ is a local complete intersection. 
If $m-1<n(t-2)$ then the same argument with exchanging $m$ and $n$, shows that $\operatorname{Tjur}^{T}(X)$ is a local complete intersection.

As we saw in Example 4.5 and Example 4.6 the theorem can still hold if $t<3$, but next we will give an example with $t=2$ where we have that $\operatorname{Tjur}_{I}(X) \neq \widetilde{\operatorname{Tjur}}_{I}(X)$ and $\operatorname{Tjur}_{J}^{T}(X) \neq{\widetilde{\operatorname{Tjur}_{J}}}^{T}(X)$ for all $I, J$. But in the example both $\operatorname{Tjur}(X)$ and $\operatorname{Tjur}^{T}(X)$ are complete intersections.

Example 5.3. Let $X \subset \mathbb{C}^{3}$ be a determinantal singularity of type $(3,2,2)$ given by

$$
F_{3}(x, y, z, w)=\left(\begin{array}{ccc}
z & y & x^{k-3} \\
0 & x & y
\end{array}\right) .
$$

For $k>4$. The $\widetilde{\operatorname{Tjur}}_{I}(X)$ is given in the three charts $I=\{1\},\{2\},\{3\}$. In the first chart the matrix is

$$
F_{\{1\}}^{\prime}\left(x, y, z, a_{2}, a_{3}\right)=\left(\begin{array}{cc}
y-a_{2} z & x^{k-3}-a_{3} z \\
x & y-a_{3} x
\end{array}\right) .
$$

We see that $\widetilde{\operatorname{Tjur}}_{\{1\}}(X)$ is the fibre over 0 (given by $x=y=z=0$ ) union the $z$-axis (given by $x=y=a_{2}=a_{3}=0$ ), so we get that $\operatorname{Tjur}_{\{1\}}(X)$ is the $z$-axis.

In the second chart we get

$$
F_{\{2\}}^{\prime}\left(x, y, z, w, a_{1}, a_{3}\right)=\left(\begin{array}{cc}
z-a_{2} y & x^{k-3}-a_{3} y \\
-a_{1} x & y-a_{3} x
\end{array}\right) .
$$

Here we see that $\widetilde{\operatorname{Tjur}}_{\{2\}}(X)$ is the fibre 0 (given by $x=y=z=0$ ) union the curve singularity given by $x^{k-4}-a_{3}^{2}=0, y=a_{3} x$ and $a_{1}=z=0$. Hence $\operatorname{Tjur}_{\{2\}}(X)$ is a $A_{k-5}$ plane curve singularity embedded in $\mathbb{C}^{5}$.

In the last chart we

$$
F_{\{3\}}^{\prime}\left(x, y, z, w, a_{1}, a_{2}\right)=\left(\begin{array}{cc}
z-a_{1} x^{k-3} & y-a_{2} x^{k-3} \\
-a_{1} y & x-a_{2} y
\end{array}\right) .
$$

Now we see that $\widetilde{\operatorname{Tjur}}_{\{2\}}(X)$ is the fibre 0 (given by $x=y=z=0$ ) union the curve given by $1-a_{2}^{2} x^{k-4}=0, y=a_{2} x^{k-3}$ and $a_{1}=z=0$. Hence $\operatorname{Tjur}_{\{2\}}(X)$ is a smooth curve in this chart.

So $\operatorname{Tjur}(X)$ is a line disjoint union a $A_{k-5}$ curve, and the fibre over 0 is 2 dimensional.

If we calculate $\widetilde{\operatorname{Tjur}}_{I}^{T}(X)$ in the charts $\{1\}$ and $\{2\}$. We get

$$
F_{\{1\}}^{\prime}\left(x, y, z, a_{2}\right)=\left(\begin{array}{c}
-a_{2} z \\
x-a_{2} y \\
y-a_{2} x^{k-3}
\end{array}\right) \text { and } F_{\{2\}}^{\prime}\left(x, y, z, w, a_{1}\right)=\left(\begin{array}{c}
z \\
y-a_{1} x \\
x^{k-3}-a_{1} y
\end{array}\right) \text {. }
$$

We see that in the first chart we have a line union the fibre over 0 and in the second chart we have an $A_{k-5}$ curve singularity union the fibre over zero.

So in this case we have that $\operatorname{Tjur}(X)$ and $\operatorname{Tjur}^{T}(X)$ are the same, a line disjoint union an $A_{k-5}$. Notice that in this case $\operatorname{Tjur}(X)$ is also local complete intersection. If we consider that $X$ is a line through the singular point of a $A_{k-4}$, we see that the transformation separates the line from the singularity, but makes the singularity somewhat worse.

In Theorem 5.2 we saw that if $t \geq 3$ then one of $\operatorname{Tjur}(X)$ or $\operatorname{Tjur}^{T}(X)$ is a local complete intersection, the case $t=1$ is not interesting, because in this case $\operatorname{Tjur}(X)=\operatorname{Tjur}^{T}(X)=X$ and $X$ is a complete intersection. The next proposition will explain the case for $t=2$. 
Proposition 5.4. Let $X$ be an EIDS of type $(m, n, 2)$, then one of $\operatorname{Tjur}(X)$ or $\operatorname{Tjur}^{T}(X)$ is a local complete intersection if $\min (n, m) \leq \operatorname{dim} X-\operatorname{dim} X^{1}$.

Proof. To prove that $X$ is a complete intersection we just need to see that $\operatorname{dim} X^{1}<$ $N-m(n-t+1)=N-m(n-1)$ by Proposition 5.1. But $(m-t+1)(n-t+1)=$ $(m-1)(n-1)=\operatorname{codim} X$, hence the inequality becomes $\operatorname{dim} X^{1}<(m-1)(n-1)+$ $\operatorname{dim} X-m(n-1)$. Hence $\operatorname{Tjur}(X)=\widetilde{\operatorname{Tjur}}(X)$ and hence a complete intersection if $n-1<\operatorname{dim} X-\operatorname{dim} X^{1}$. The case $\operatorname{Tjur}^{T}(X)=\widetilde{\operatorname{Tjur}^{T}}(X)$ is gotten by exchanging $n$ and $m$ and the result follows.

Corollary 5.5. Let $X$ be an EIDS of type $(m, n, 2)$ with an isolated singularity, then one of $\operatorname{Tjur}(X)$ or $\operatorname{Tjur}^{T}(X)$ is a local complete intersection if $\min (n, m) \leq \operatorname{dim} X$.

These result is only in the one direction, because what we really prove is that if

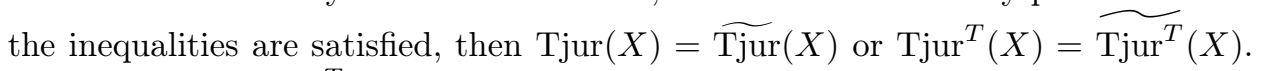
$\operatorname{But} \operatorname{Tjur}(X)$ or $\operatorname{Tjur}^{T}(X)$ could still be local complete intersections, even if this is not true.

\section{Using Tuurina Transform to Resolve Hypersurface Singularities}

In the previous section we saw that very often the Tjurina transform is a complete intersection, which means that one can not get a resolution by using only the Tjurina transform. Notice also that in several of the examples $\operatorname{Tjur}(X)$ is normal, so using only Tjurina transform and normalizations will also not produce a resolution. In the next example we will look at the case of the $A_{n}$ surface singularities and see that it might not be completely impossible to use the Tjurina transform to achieve a resolution.

Example 6.1 ( $A_{n}$ singularities). We will in this example see how different representations of the simple $A_{n}$ singularity can lead to different Tjurina transforms.

First we can of course represent $A_{n}$ as a determinantal singularity of type $(1,1,1)$, then the Tjurina transform of $A_{n}$ is just $A_{n}$ itself, by Proposition [5, But we can also represent $A_{n}$ as a determinantal singularity of type $(2,2,2)$ like the following:

$$
F(x, y, z)=\left(\begin{array}{cc}
x & z^{l} \\
z^{n-l+1} & y
\end{array}\right)
$$

where $0<l \leq n$. In this case we get that the Tjurina transform is given by:

$$
F_{\{1\}}^{\prime}\left(x, y, z, a_{2}\right)=\left(\begin{array}{c}
z^{l}-a_{2} x \\
y-a_{2} z^{n-l+1}
\end{array}\right) \text { and } F_{\{2\}}^{\prime}\left(x, y, z, a_{1}\right)=\left(\begin{array}{c}
x-a_{1} z^{l} \\
z^{n-l+1}-a_{1} y
\end{array}\right) \text {. }
$$

So we see that $\operatorname{Tjur}\left(A_{n}\right)$ using this representations have an $A_{l-1}$ and an $A_{n-l}$ singularity, so we have simplified the singularity. It is clear, that by writing these new $A_{m}$ singularities as determinantal singularities of type $(2,2,2)$, we can apply the Tjurina transform again to simplify the singularity. By repeatedly doing this we can resolve the $A_{n}$ singularity.

As we can see in Example 6.1 the Tjurina transform depends not only on the singularity type of $X$ but we also get different transforms if we have different matrix presentations of the same type.

We will in the next example show how to obtain a resolution trough repeated Tjurina transform changing the determinantal type and matrix presentation. By this we mean that the Tjurina transform gives us a complete intersection of the form $(m, n, 1)$, which by change of coordinates locally can be seen as a hypersurface. We will then write this hypersurface as a determinantal singularity of type $(t, t, t)$. 
Example 6.2 ( $E_{7}$ singularity). The simple surface singularity $E_{7}$ can be defined by the following equation $y^{2}+x\left(x+z^{3}\right)=0$. This can be seen as a determinantal singularity of type $(2,2,2)$ given by the following matrix: $\left(\begin{array}{cc}y & x^{2}+z^{3} \\ -x & y\end{array}\right)$. We then perform the Tjurina transform and get:

$$
F_{\{1\}}^{\prime}\left(x, y, z, a_{2}\right)=\left(\begin{array}{c}
x^{2}+z^{3}-a_{2} x \\
y-a_{2} x
\end{array}\right) \text { and } F_{\{2\}}^{\prime}\left(x, y, z, a_{1}\right)=\left(\begin{array}{c}
y-a_{1}\left(x^{2}+z^{3}\right) \\
-x-a_{1} y
\end{array}\right) .
$$

By changing coordinates we see that $F_{\{1\}}^{\prime}$ is equivalent to the hypersurface $x^{2}+$ $z^{3}+w^{2} x=0$, which has a singular point at $(0,0,0)$, and $F_{\{2\}}^{\prime}$ is equivalent to the hypersurface $x+v^{2}\left(x^{2}+z^{3}\right)=0$ which is non singular.

So we will continue working in the first chart, and we will denote this singularity $\operatorname{Tjur}\left(E_{7}\right)$. In the coordinates $x^{2}+z^{3}+w^{2} x=0$ the exceptional divisor $E_{1}=$ $\left(\pi^{T j}\right)^{-1}(0)$ is given by $x=z=0$. We now write $\operatorname{Tjur}\left(E_{7}\right)$ as the matrix $\left(\begin{array}{cc}x & z^{2} \\ -z x+w^{2}\end{array}\right)$ and perform the Tjurina transform.

$$
F_{\{1\}}^{\prime}\left(x, z, w, a_{2}\right)=\left(\begin{array}{c}
z^{2}-a_{2} x \\
x+w^{2}+a_{2} z
\end{array}\right) \text { and } F_{\{2\}}^{\prime}\left(x, z, w, a_{1}\right)=\left(\begin{array}{c}
x-a_{1} z^{2} \\
-z-a_{1}\left(x+w^{2}\right)
\end{array}\right) .
$$

The first chart is equivalent to the hypersurface $z^{2}+y w^{2}+y^{2} z=0$ which has a singularity at $(0,0,0)$, and the second chart is equivalent to $z+v\left(v z^{2}+w^{2}\right)=0$ which is smooth. The exceptional divisor consist of two components the strict transform of exceptional divisor from before (which we by abuse of notation denote by $E_{1}$ ) is given by $z=y=0$ and the new addition $E_{2}$ given by $x=w=0$. They intersect each other in the singular point.

We will continue in the first chart and denote this singularity by $\operatorname{Tjur}^{2}\left(E_{7}\right)$. It can be given by the matrix $\left(\begin{array}{cc}y & z \\ -z y z+w^{2}\end{array}\right)$ as a determinantal singularity of type $(2,2,2)$. The Tjurina transform is

$$
F_{\{1\}}^{\prime}\left(y, z, w, a_{2}\right)=\left(\begin{array}{c}
z-a_{2} y \\
y z+w^{2}+a_{2} z
\end{array}\right) \text { and } F_{\{2\}}^{\prime}\left(y, z, w, a_{1}\right)=\left(\begin{array}{c}
y-a_{1} z \\
-z-a_{1}\left(y z+w^{2}\right)
\end{array}\right) .
$$

I the first chat we have the hypersurface $x y^{2}+w^{2}+x^{2} y=0$ which has $(0,0,0)$ as its only singular point. The second chart is $z+v\left(v z^{2}+w^{2}\right)=0$ which is smooth. The exceptional divisor consist of $E_{1}$ given by $z=v=0$ (so it only exists in the second chart), $E_{2}$ given by $x=w=0$ and the new $E_{3}$ given by $y=w=0 . E_{1}$ and $E_{2}$ does not meet, but $E_{3}$ intersects them both, $E_{1}$ in a smooth point and $E_{2}$ in the singular point.

We represent the singularity $\operatorname{Tjur}^{3}\left(E_{7}\right)$ as the matrix $\left(\begin{array}{cc}x y & w \\ -w & x+y\end{array}\right)$. The Tjurina transform is then

$$
F_{\{1\}}^{\prime}\left(x, y, w, a_{2}\right)=\left(\begin{array}{c}
w-a_{2} x y \\
x+y+a_{2} w
\end{array}\right) \text { and } F_{\{2\}}^{\prime}\left(x, y, w, a_{1}\right)=\left(\begin{array}{c}
x y-a_{1} w \\
-w-a_{1}(x+y)
\end{array}\right) .
$$

In the first chart we have the hypersurface $x+y+v^{2} x y=0$ which is smooth. The second chart gives the hypersurface singularity $x y+z^{2}(x+y)=0$, which has a singular point at $(0,0,0) . E_{1}$ does not exists in $\operatorname{Tjur}^{3}\left(E_{7}\right)$, but intersects $E_{3}$ in a smooth point in the other charts. $E_{2}$ is given by $x=z=0, E_{3}$ is given by $y=z=0$ and the new $E_{4}$ is given by $x=y=0 . E_{2}, E_{3}$ and $E_{4}$ intersect each other in the singular point.

Next we can represent the singularity $\operatorname{Tjur}^{4}\left(E_{7}\right)$ by the matrix $\left(\begin{array}{cc}x & z(x+y) \\ -z & y\end{array}\right)$. The Tjurina transform is then

$$
F_{\{1\}}^{\prime}\left(x, y, z, a_{2}\right)=\left(\begin{array}{c}
z(x+y)-a_{2} x \\
y+a_{2} z
\end{array}\right) \text { and } F_{\{2\}}^{\prime}\left(x, y, z, a_{1}\right)=\left(\begin{array}{c}
x-a_{1} z(x+y) \\
-z-a_{1} y
\end{array}\right) .
$$


The first chart gives the hypersurface $z x-w x-w z^{2}=0$ which has a singular point at $(0,0,0)$, and the second chart gives $x-v^{2} y(x+y)=0$ which is smooth. The exceptional divisor consist of $E_{2}$ given by $z=v=0$ so not in the chart that contains the singularity, $E_{3}$ given by $z=w=0, E_{4}$ given by $x=w=0$ and $E_{5}$ given by $X=z=0$. $E_{2}$ intersects $E_{5}$ in a smooth point, $E_{3}, E_{4}$ and $E_{5}$ intersect each other in the singular point, and $E_{3}$ intersects $E_{1}$ in a smooth point outside these charts.

We can represent $\operatorname{Tjur}^{5}\left(E_{7}\right)$ by the matrix $\left(\begin{array}{cc}z & x \\ w & x-w z\end{array}\right)$. In this case the Tjurina transform is

$$
F_{\{1\}}^{\prime}\left(x, z, w, a_{2}\right)=\left(\begin{array}{c}
x-a_{2} z \\
x-w z-a_{2} w
\end{array}\right) \text { and } F_{\{2\}}^{\prime}\left(x, z, w, a_{1}\right)=\left(\begin{array}{c}
z-a_{1} x \\
w-a_{1}(x-w z)
\end{array}\right) .
$$

The first chart gives the hypersurface $y z-w z+y w=0$ which has a singularity at $(0,0,0)$, and the second chart gives the smooth hypersurface $w-v x-v^{2} w x=0$. The exceptional divisor consists of $E_{1}$ and $E_{2}$ that do not appear in any of these charts, $E_{3}$ given by $z=v=0$ (so only appearing in the second chart), $E_{4}$ given by $w=y=0, E_{5}$ given by $z=y=0$ and $E_{6}$ given by $w=z=0 . E_{3}$ intersects $E_{1}$ and $E_{6}$ in different smooth points, $E_{2}$ intersects $E_{5}$ in a smooth point, $E_{4}, E_{5}$ and $E_{6}$ intersect each other in the singular point.

For $\operatorname{Tjur}^{6}\left(E_{7}\right)$ we use the matrix $\left(\begin{array}{cc}y & w \\ z & z+w\end{array}\right)$. We get that the Tjurina transform is

$$
F_{\{1\}}^{\prime}\left(y, z, w, a_{2}\right)=\left(\begin{array}{c}
w-a_{2} y \\
z+w-a_{2} z
\end{array}\right) \text { and } F_{\{2\}}^{\prime}\left(y, z, w, a_{1}\right)=\left(\begin{array}{c}
y-a_{1} w \\
z-a_{1}(z+w)
\end{array}\right) .
$$

The first chart is the smooth hypersurface $z+x y-x z=0$, and the second chart is $z-v z-y=0$ which is also smooth. So we have reach a resolution of $E_{7}$. The exceptional divisors consist of $E_{1}, \ldots, E_{7}$, where only $E_{4} \ldots, E_{7}$ appears in the last two charts. $E_{4}$ is given by $y=x-1=0, E_{5}$ is given by $z=v=0, E_{6}$ is given by $z=x=0$ and $E_{7}$ is given by $z=y=0 . E_{7}$ intersects $E_{4}, E_{5}$ and $E_{6}$ in three different smooth points, $E_{2}$ intersects $E_{5}$ in a smooth point, and $E_{3}$ intersects $E_{1}$ and $E_{6}$ in two different smooth points. If we represent the exceptional divisor by a dual resolution graph (where vertices represent the curves and edges represents the intersection points) we get:

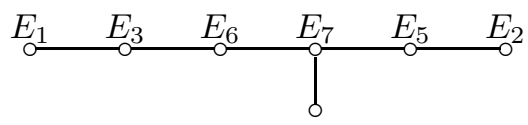

which is indeed the $E_{7}$ graph.

$E_{4}$

One can also use this method to produce resolutions of the $D_{n}$ and $E_{6}$ singularities, and probably many more. But it is not always possible to use this method, the $E_{8}$ given by $x^{2}+y^{3}+z^{5}$ can not be written as a the determinant of a $2 \times 2$ matrix which is 0 at the origin of $\mathbb{C}^{3}$, nor can it be written as the determinant of a larger matrix such the value at the origin is 0 . If the value at the origin is not zero, then the Tjurina transform does not improve the singularity, it only changes variables.

\section{REFERENCES}

[ACGH85] E. Arbarello, M. Cornalba, P. A. Griffiths, and J. Harris. Geometry of algebraic curves. Vol. I, volume 267 of Grundlehren der Mathematischen Wissenschaften [Fundamental Principles of Mathematical Sciences]. Springer-Verlag, New York, 1985.

[DP14] James Damon and Brian Pike. Solvable groups, free divisors and nonisolated matrix singularities II: Vanishing topology. Geom. Topol., 18(2):911-962, 2014.

[FZ15] A. Fruehbis-Krueger and M. Zach. On the Vanishing Topology of Isolated CohenMacaulay Codimension 2 Singularities. ArXiv e-prints, January 2015. 
ON TJURINA TRANSFORM AND RESOLUTION OF DETERMINANTAL SINGULARITIES 16

[GR15] T. Gaffney and A. Rangachev. Pairs of modules and determinantal isolated singularities. ArXiv e-prints, December 2015.

[GZĖ09] S. M. Guseĭn-Zade and V. Ėbeling. On the indices of 1-forms on determinantal singularities. Tr. Mat. Inst. Steklova, 267(Osobennosti i Prilozheniya):119-131, 2009.

[Mil68] John Milnor. Singular points of complex hypersurfaces. Annals of Mathematics Studies, No. 61. Princeton University Press, Princeton, N.J., 1968.

[NBOOT13] J. J. Nuño-Ballesteros, B. Oréfice-Okamoto, and J. N. Tomazella. The vanishing Euler characteristic of an isolated determinantal singularity. Israel J. Math., 197(1):475495, 2013.

[Spi90] Mark Spivakovsky. Sandwiched singularities and desingularization of surfaces by normalized Nash transformations. Ann. of Math. (2), 131(3):411-491, 1990.

[SRDSP14] Maria Aparecida Soares Ruas and Miriam Da Silva Pereira. Codimension two determinantal varieties with isolated singularities. Math. Scand., 115(2):161-172, 2014.

[Tju68] G. N. Tjurina. Absolute isolation of rational singularities, and triple rational points. Funkcional. Anal. i Priložen., 2(4):70-81, 1968.

[vS87] Duco van Straten. Weakly Normal Surface Singularities and Their Improvements. $\mathrm{PhD}$ thesis, Universiteit Leiden, 1987.

\section{ICMC-USP}

E-mail address: helge@imf.au.dk 